\title{
Serum potassium dynamics during acute heart failure hospitalization
}

Pedro Caravaca Perez ${ }^{1,2}$, José R. González-Juanatey ${ }^{1,3}$, Jorge Nuche ${ }^{1,4}$, Laura Morán Fernández ${ }^{1,2}$, David Lora Pablos ${ }^{5}$, Jesús Alvarez-García ${ }^{1,6}$, Ramón Bascompte Claret ${ }^{7}$, Manuel Martínez Selles ${ }^{1,8}$, Rafael Vázquez García ${ }^{9}$, Luis Martínez Dolz ${ }^{1,10}$, Marta Cobo-Marcos $^{1,11}$, Domingo Pascual Figal ${ }^{1,12}$, Maria G. Crespo-Leiro ${ }^{1,13}$, Julio Nuñez Villota $^{1,14}$, Juan Cinca Cuscullola ${ }^{4} \&$ Juan F. Delgado ${ }^{1,2,15}$

CIBER de Enfermedades Cardiovasculares (CIBERCV),Madrid, Spain

2 Department of Cardiology, Hospital Universitario 12 de Octubre, Instituto de Investigación Sanitaria Hospital 12 CIBER de Enfermedades Cardiovasculares (CIBERCV) Madrid, Spain

3 Department of Cardiology, Complejo Hospitalario Universitario de Santiago de Compostela, Facultad de Medicina, Universidad de Santiago, Santiago de Compostela, Spain

4 Centro Nacional de Investigaciones Cardiovasculares, Madrid, Spain

5 Instituto de Investigación Sanitaria Hospital 12 Octubre (imas12), Universidad Complutense de Madrid, CIBERESP, Madrid, Spain

6 Department of Cardiology, Hospital Universitario Santa Creu i Sant Pau, Universidad de Barcelona, Barcelona, Spain

7 Hospital Universitari Arnau de Vilanova, IBRLLEIDA, Lleida, Spain

8 Department of Cardiology, Hospital Universitario Gregorio Marañón, Instituto de Investigación Sanitaria IiGM, Universidad Europea, Madrid, Spain

9 UGC de Cardiología, Hospital Universitario Puerta del Mar, Cádiz, Spain

10 Department of Cardiology, Hospital Universitario Y Politécnico La Fe, IIS La Fe, Valencia, Spain

11 Department of Cardiology, Hospital Universitario Puerta de Hierro, Madrid, Spain

12 Department of Cardiology, Hospital Universitario Virgen de La Arrixaca, Universidad de Murcia, Murcia, Spain

13 Department of Cardiology, Complexo Hospitalario Universitario A Coruña (CHUAC), Instituto de Investigación Biomedica A Coruña (INIBIC), Universidade da Coruña (UDC), A Coruña, Spain

14 Servicio de Cardiología, Hospital Clinico Universitario de Valencia, INCLIVA, Universitat de Valencia, Valencia, Spain

15 Facultad de Medicina, Universidad Complutense de Madrid (UCM), Madrid, Spain

\section{Abstract}

Background

Available information about prognostic implications of potassium levels alteration in the setting of acute heart failure $(\mathrm{AHF})$ is scarce.

Objectives

We aim to describe the prevalence of dyskalemia (hypo or hyperkalemia), its dynamic changes during AHFhospitalization, and its long-term clinical impact after hospitalization. 
We analyzed 1779 patients hospitalized with AHF who were included in the REDINSCOR II registry. Patients were classified in three groups, according to potassium levels both on admission and discharge: hypokalemia (potassium $<3.5 \mathrm{mEq} / \mathrm{L}$ ), normokalemia (potassium $=3.5-5.0 \mathrm{mEq} / \mathrm{L}$ and, hyperkalemia (potassium $>5 \mathrm{mEq} / \mathrm{L}$ ).

Results

The prevalence of hypokalemia and hyperkalemia on admission was 8.2 and $4.6 \%$, respectively, and 6.4 and $2.7 \%$ at discharge. Hyperkalemia on admission was associated with higher in-hospital mortality $(\mathrm{OR}=2.32[95 \%$ CI: 1.04-5.21] $\mathrm{p}=0.045)$. Among patients with hypokalemia on admission, 79\% had normalized potassium levels at discharge. In the case of patients with hyperkalemia on admission, $89 \%$ normalized kalemia before discharge. In multivariate Cox regression, dyskalemia was associated with higher 12-month mortality, (HR $=1.48$ [95\% CI, 1.12 1.96], $\mathrm{p}=0.005$ ). Among all patterns of dyskalemia persistent hypokalemia (HR $=3.17$ [95\% CI: 1.71-5.88]; $\mathrm{p}<0.001)$, and transient hyperkalemia $(\mathrm{HR}=1.75$ [95\% CI: $1.07-2.86] ; \mathrm{p}=0.023)$ were related to reduced 12-month survival.

\section{Conclusions}

Potassium levels alterations are frequent and show a dynamic behavior during AHF admission. Hyperkalemia on admission is an independent predictor of higher in-hospital mortality. Furthermore, persistent hypokalemia and transient hyperkalemia on admission are independent predictors of 12-month mortality.

\section{Introduction}

Heart failure (HF) is the ultimate consequence of a variety of cardiac conditions, and its incidence and prevalence are continuously growing [1]. Its clinical behavior is characterized by a progressive deterioration of affected patients with an increased number of decompensations requiring hospitalization [2]. Despite the continuous development of new HF therapies, this syndrome remains related to a low long-term survival estimated as 50\% in the fifth year after diagnosis [3].

Potassium plays a pivotal role in cellular homeostasis, and it is involved in numerous physiological processes. Serum potassium levels disturbances are frequent in the setting of acute heart failure (AHF), due to neurohormonal disorders, the presence of chronic comorbidities (i.e., chronic kidney disease, diabetes mellitus), and that induced by specific treatment such as diuretics and renin-angiotensinaldosterone system (RAAS) inhibitors [4]. The presence of dyskalemia may prevent physicians from the initiation or titration of proved beneficial treatments [5], and it also increases the risk of arrhythmic complications [4].

The presence of potassium level disturbances has shown to negatively influence the prognosis of chronic HF patients [6]. However, information about the prevalence of dyskalemia and dynamic changes of potassium levels in the setting of AHF is limited to selected populations, without available data of a real-life cohort. Besides, the short and long-term prognostic implications of these disturbances during AHF hospitalization are not well defined, with controversial results previously published $[7,8,9]$.

This analysis aims to determine the prevalence of dyskalemia, the evolution of potassium levels during admission, and its implication in short and long-term survival in a contemporary cohort of patients admitted due to AHF. 


\section{Methods}

\section{Study population}

REDINSCOR registry (from Spanish "Red de investigación clínica y básica en insuficiencia cardiaca") is a prospective and multicentric registry completed from October 2013 and December 2014 in 20 university hospitals in Spain. A total of 1.831 patients were included. All patients were aged $\geq 18$ years and had signs and symptoms of AHF requiring in-hospital management. Both patients with first admission and re-admissions were eligible for inclusion. The registry included patients with reduced left ventricular ejection fraction $(\mathrm{LVEF})(<40 \%)$, preserved LVEF $(\geq 50 \%)$, and intermediate LVEF $(40-$ $49 \%$ ). Diagnosis and treatment of every patient were supervised by a cardiology specialist and was performed according to current clinical practice guidelines [3]. Patients with AHF in the setting of an STsegment elevation acute coronary syndrome, those with a vital prognosis shorter than 1 year, and those without the possibility of long-term outpatient follow-up were not included.

For this substudy, only patients with serum potassium levels quantified on admission and immediately before discharge were included.

The ethics committee approved the REDINSCOR II registry protocol from every participant center, and all patients signed informed consent before inclusion.

\section{Variables}

All clinical, laboratory-derived, electrocardiographic, and echocardiographic parameters were recorded during admission. Depending on kalemia, patients were classified into 3 categories: 1) hyperkalemia (potassium $>5 \mathrm{mEq} / \mathrm{L}$ ); normokalemia (potassium $=3.5-5 \mathrm{mEq} / \mathrm{L}$ ); and hypokalemia (potassium $<3.5 \mathrm{mEq} / \mathrm{L}$ ). Changes in serum potassium levels were established as the difference between discharge levels and admission levels $(\mathrm{mEq} / \mathrm{L})$. Changes between these kalemia-based categories from admission to discharge were also analyzed.

Glomerular filtration rate (GFR) was estimated with the chronic kidney disease-Epidemiology Collaboration (CKD-EPI) formula. Chronic kidney disease was defined as a GFR $<60 \mathrm{~mL} / \mathrm{min} / 1.73 \mathrm{~m}^{2}$.

\section{Event definition}

The primary outcome was all-cause mortality during admission and at a 1-year follow-up. After discharge, every patient was enrolled in a follow-up protocol, which included clinical evaluation at 1,3 , 6 , and 12 months.

\section{Statistical analysis}

Clinical variables were summarized for every kalemia-based category with comparisons between them.

Quantitative variables were expressed as mean (standard deviation) or median $\left(\mathrm{Q}_{1}-\mathrm{Q}_{2}\right)$ when appropriate. Categorical variables are defined as frequency (\%). Comparisons were made using the $\chi^{2}$ test or exact Fisher's test for categorical variables and ANOVA, Kruskal-Wallis test, Student's " $t$ " test, or Mann-Whitney "U" test for continuous variables. A multivariate logistic regression analysis was performed to determine clinical predictors hyperkalemia or hypokalemia on admission. 
The association between potassium levels and in-hospital mortality was evaluated through a fractional polynomial analysis. A multivariate logistic regression analysis was performed to assess the association between the three categories of potassium on admission (hypokalemia, normal kalemia, and hyperkalemia) with in-hospital mortality. Variables with a univariate association with in-hospital mortality with a $p$ value of $<0.1$ were included in the initial model. Variables were removed from the initial model with the stepwise backward method with a cutoff point of $p<0.05$. The discrimination capacity of the final model was calculated by analyzing the area under the curve, whereas calibration was assessed using the Hosmer-Lemeshow goodness of fit test.

Kaplan-Meier method was used to evaluate the association between the different patterns of dyskalemia with 12-month mortality. Survival curves were compared using the log-rank test. A Cox regression analysis was performed to identify the predictors of mortality during the 12-month follow-up. Variables with univariate association with mortality with a $\mathrm{p}$ value $<0.1$ were included in the initial model. Variables were removed from the model with the stepwise backward method with a cutoff point of $p<0.05$. The proportional risk assumption was evaluated using the Schoenfeld residual test. The discriminatory capacity of the models was estimated using the Harrell " $\mathrm{C}$ " statistic and its calibration by the Groennesby and Borgan test.

Missing values were imputed using the multivariate imputation package ("chained equations") whenever necessary $(n=5)[10,11]$. A two-sided $p$ value $<0.05$ was considered for signification.

Data were analyzed using the statistic software Stata 15 (StataCorp. 2017. Stata Statistical Software: Release 15. College Station, TX: StataCorp LLC).

\section{Results}

At the end of the enrolment period, 1831 patients were included in the REDINSCOR registry. In 52 (2.8\%), kalemia on admission was not recorded. Thus, 1779 (97.2\%) patients were finally included in the in-hospital analysis. During hospitalization, $69(3.8 \%)$ patients died, and $26(1.45 \%)$ patients did not have potassium measure at discharge, so $1.684(91.9 \%)$ patients were included in the 1-year analysis. At 1 year after admission, 401 (22,5\%) had died (Fig. 1).

\section{Baseline characteristics}

The mean age at inclusion was $72 \pm 12$ years, with $889(50 \%)$ of included patients being older than 75 years. Men represented $59 \%$ of the patients.

Reduced, intermediate, and preserved LVEF was present in 564 (31\%), 284 (16\%), and 931 (52\%) patients, respectively. In $742(41.7 \%)$ patients, the index event was the first AHF-related admission, and in 1037 patients $(58.3 \%)$, it was a re-admission due to decompensated chronic heart failure.

Mean and median potassium levels on admission were $4.32 \pm 0.7 \mathrm{mEq} / \mathrm{L}$ and $4(3-5) \mathrm{mEq} / \mathrm{L}$, respectively. The majority of patients (1553 [87.3\%]) presented normal potassium levels on admission. On the contrary, $145(8.2 \%)$ patients had hypokalemia on admission, and hyperkalemia was present in 81 (4.6\%) (Fig. 2a). Baseline characteristics, according to serum potassium levels on admission, are detailed in Table 1.

Before discharge, mean and median kalemia were $4.3 \pm 0.71 \mathrm{mEq} / \mathrm{L}$ and $4 \mathrm{mEq} / \mathrm{L}(3-5 \mathrm{mEq} / \mathrm{L})$, respectively. The number of patients with normal potassium levels at discharge was $1569(90.9 \%)$. Among patients with dyskalemia, $111(6.4 \%)$ presented hypokalemia, and 47 (2.7\%) hyperkalemia. Baseline characteristics, according to kalemia before discharge, are summarized in Table 2. 


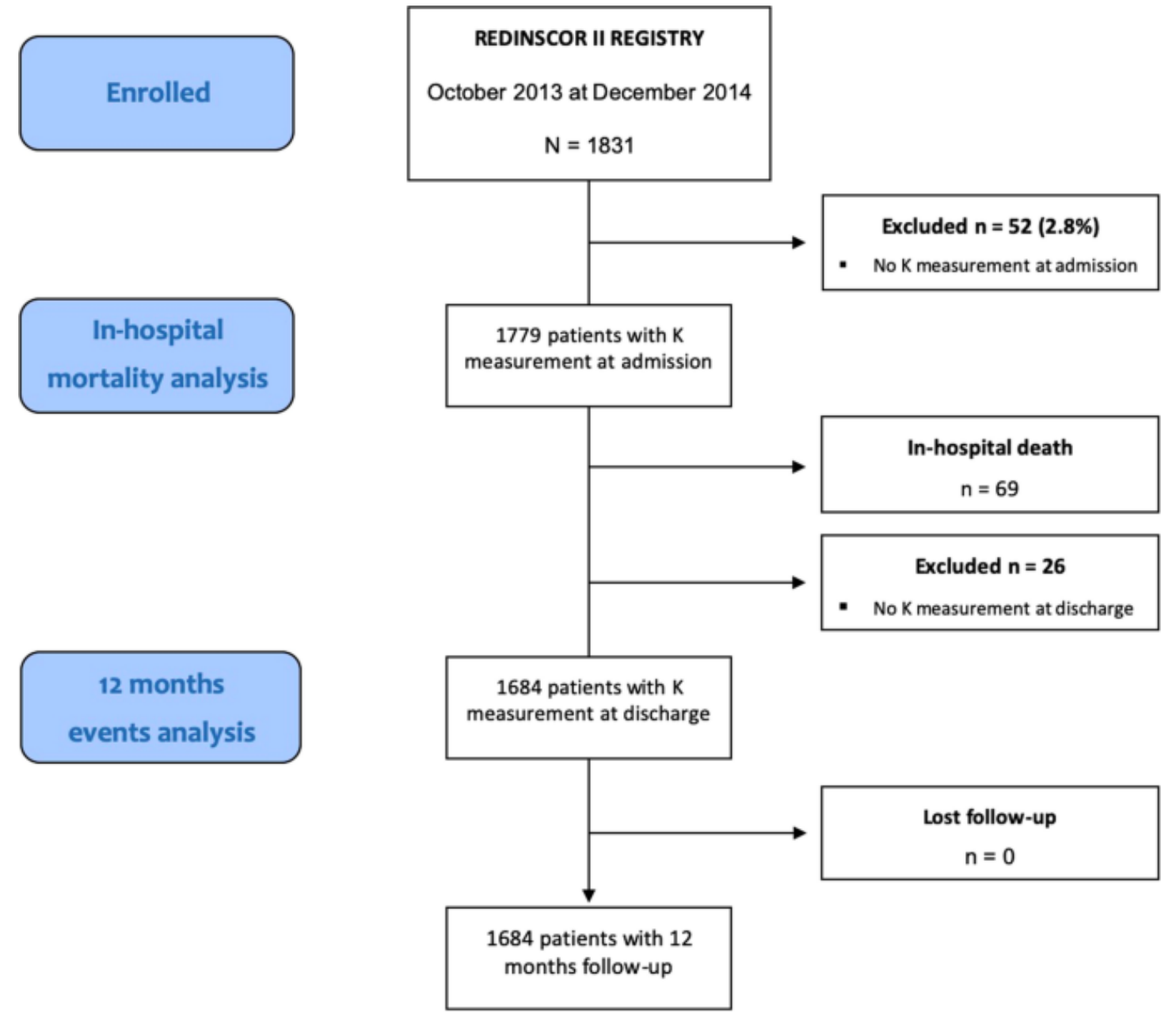

Fig. 1 Study flowchart

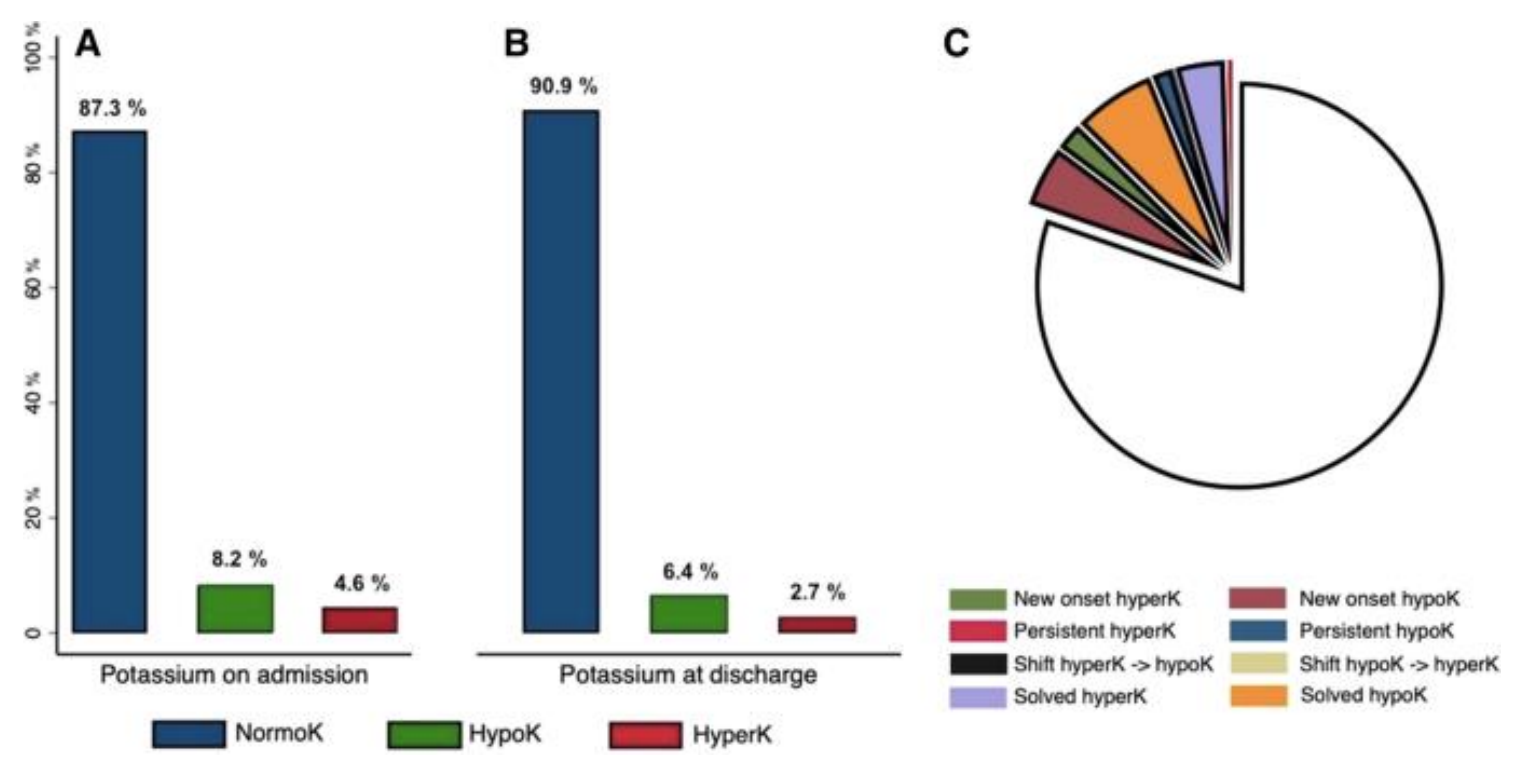

Fig.2. a Frequency of normokalemia, hypokalemia, and hyperkalemia on admission; b frequency of normokalemia, hypokalemia, and hyperkalemia before discharge; $c$ dynamic behavior of serum potassium levels 
Table 1. Baseline characteristics according to serum potassium levels on admission

\begin{tabular}{|c|c|c|c|c|c|c|c|c|c|c|c|}
\hline & & \multicolumn{2}{|c|}{ Total $(\mathrm{N}=1779)$} & \multicolumn{2}{|c|}{$\begin{array}{l}\mathrm{K}^{+}<3.5 \mathrm{mEq} / \mathrm{L} \\
(\mathrm{n}=145)\end{array}$} & \multicolumn{2}{|c|}{$\begin{array}{l}\mathrm{K}^{+} 3.5-5.0 \mathrm{mEq} / \mathrm{L} \\
(\mathrm{n}=1553)\end{array}$} & \multicolumn{2}{|c|}{$\begin{array}{l}\mathrm{K}^{+}>5.0 \mathrm{mEq} / \mathrm{L} \\
(\mathrm{n}=81)\end{array}$} & \multicolumn{2}{|c|}{$\mathrm{p}$ value } \\
\hline \multicolumn{2}{|l|}{ Age (years) } & \multicolumn{2}{|c|}{$72 \pm 12$} & \multicolumn{2}{|c|}{$72 \pm 12$} & \multicolumn{2}{|c|}{$72 \pm 12$} & \multicolumn{2}{|c|}{$74 \pm 11$} & \multicolumn{2}{|l|}{0.3} \\
\hline \multicolumn{2}{|l|}{ Age $\geq 75$ years } & \multicolumn{2}{|c|}{$890(50 \%)$} & \multicolumn{2}{|l|}{$68(47 \%)$} & \multicolumn{2}{|c|}{$778(50 \%)$} & \multicolumn{2}{|l|}{$44(54 \%)$} & \multicolumn{2}{|l|}{0.6} \\
\hline \multicolumn{2}{|l|}{ Male $(\%)$} & \multicolumn{2}{|c|}{$1046(59 \%)$} & \multicolumn{2}{|l|}{$83(57 \%)$} & \multicolumn{2}{|c|}{$916(59 \%)$} & \multicolumn{2}{|l|}{$47(58 \%)$} & \multicolumn{2}{|l|}{0.9} \\
\hline \multicolumn{2}{|l|}{ Systolic pressure $(\mathrm{mmHg})$} & \multicolumn{2}{|c|}{$134.5 \pm 29$} & $135.9 \pm 30.9$ & & 134.6 & 28.6 & $130.3 \pm 34.6$ & & 0.36 & \\
\hline Diastolic pressure $(\mathrm{mmHg}$ & & $76 \pm 18$ & & $75.7 \pm 18.2$ & & $76.2 \pm$ & 7.6 & $71.9 \pm 17.1$ & & 0.09 & \\
\hline Heart rate $(\mathrm{bpm})$ & & $88.9 \pm 2$ & 26.1 & $88.2 \pm 26.5$ & & $89.3 \pm$ & 26.2 & $82.8 \pm 22.6$ & & 0.08 & \\
\hline $\mathrm{CV}$ risk factors & & & & & & & & & & & \\
\hline Hypertension (\%) & & $1378(7$ & & $110(77)$ & & 1196( & & $72(89)$ & & 0.06 & \\
\hline Diabetes mellitus (\%) & & $829(47)$ & & $71(49)$ & & $711(4$ & & $47(59)$ & & 0.06 & \\
\hline Smoking habit $(\%)$ & & $186(10$ & & $13(9)$ & & $170(1$ & & $3(4)$ & & 0.09 & \\
\hline Charlson index (score) & & $3 \pm 3$ & & $4 \pm 3$ & & $3 \pm 3$ & & $5 \pm 3$ & & $<0.001$ & \\
\hline Barthel index (score) & & $89 \pm 21$ & & $87 \pm 22$ & & $90 \pm 2$ & & $86 \pm 22$ & & 0.05 & \\
\hline $\operatorname{LVEF}(\%)$ & & $45 \pm 18$ & & $44 \pm 17$ & & $45 \pm 1$ & & $47 \pm 18$ & & 0.6 & \\
\hline $\mathrm{LVEF}<40 \%(\%)$ & & $564(41)$ & & $51(46)$ & & $484(4$ & & $29(41)$ & & 0.4 & \\
\hline Ischemic heart disease (\%) & & $605(39$ & & $44(34)$ & & $527(3$ & & $34(45)$ & & 0.3 & \\
\hline Atrial fibrillation (\%) & & $682(40$ & & $62(46)$ & & $593(4$ & & $27(34)$ & & 0.2 & \\
\hline Laboratory parameters & & & & & & & & & & & \\
\hline Hemoglobin (g/dL) & & $123 \pm 21$ & & $124 \pm 18$ & & $123 \pm$ & & $116 \pm 24$ & & 0.05 & \\
\hline Creatinin (mg/dL) & & $1.14 \pm 0$ & .62 & $1.09 \pm 0.57$ & & $1.12 \pm$ & .60 & $1.63 \pm 0.70$ & & $<0.001$ & \\
\hline GFR (CKD-EPI) (mL/min & $\left./ 1.73 \mathrm{~m}^{2}\right)$ & $60 \pm 25$ & & $62 \pm 24$ & & $61 \pm 2$ & & $38 \pm 18$ & & $<0.001$ & \\
\hline GFR $($ CKD-EPI $)<60(\mathrm{mI}$ & $\left./ \mathrm{min} / 1.73 \mathrm{~m}^{2}\right)$ & $930(52)$ & & $68(47)$ & & $793(5$ & & $69(85)$ & & $<0.001$ & \\
\hline GFR $($ CKD-EPI $)<30(\mathrm{mI}$ & $\left./ \mathrm{min} / 1.73 \mathrm{~m}^{2}\right)$ & $218(12$ & & $6(4)$ & & $78(5)$ & & $13(16)$ & & $<0.001$ & \\
\hline Sodium $(\mathrm{mEq} / \mathrm{L})$ & & $139 \pm 5$ & & $139 \pm 6$ & & $139 \pm$ & & $136 \pm 6$ & & $<0.001$ & \\
\hline Potassium on admission & $\mathrm{mEq} / \mathrm{L})$ & $4.3 \pm 0.7$ & & $3.0 \pm 0.1$ & & $4.3 \pm 0$ & & $6.1 \pm 0.5$ & & $<0.001$ & \\
\hline Potassium at discharge $(\mathrm{m}$ & $\mathrm{Eq} / \mathrm{L})$ & $4.3 \pm 0.7$ & & $4.0 \pm 0.7$ & & $4.3 \pm 0$ & & $4.5 \pm 0.6$ & & $<0.001$ & \\
\hline NT-ProBNP on admission & $(\mathrm{pg} / \mathrm{mL})$ & $6706 \pm 7$ & 7716 & $7916 \pm 8997$ & & $6486 \pm$ & 7502 & $8457 \pm 8214$ & & 0.02 & \\
\hline NT-ProBNP at discharge & $(\mathrm{pg} / \mathrm{mL})$ & $4920 \pm 2$ & 2408 & $4163 \pm 5047$ & & $4921 \pm$ & 7759 & $6024 \pm 6823$ & & 0.01 & \\
\hline Treatment & Admission & Discharge & Admission & n Discharge & Adr & nission & Discharge & Admission & Disc & charge & \\
\hline ACEI/ARB & $1075(60)$ & $1195(67)$ & $88(61)$ & $94(65)$ & 923 & $(59)$ & $1050(67)$ & $64(79)$ & 51( & $(63)$ & 0.001 \\
\hline$\beta$-Blockers & $966(54)$ & $1234(71)$ & $89(61)$ & $101(74)$ & 825 & (53) & $1079(71)$ & $52(64)$ & 54( & (73) & 0.03 \\
\hline MRA & $426(24)$ & $773(45)$ & $35(24)$ & $67(50)$ & 355 & (23) & $678(45)$ & $36(44)$ & 28( & (38) & $<0.001$ \\
\hline Digoxin & $195(11)$ & $298(18)$ & $24(17)$ & $31(23)$ & 157 & (10) & $250(17)$ & $14(17)$ & 17( & (23) & 0.01 \\
\hline Ivabradin & $86(5)$ & $129(8)$ & $9(6)$ & $10(7)$ & 73 & & $110(7)$ & $4(5)$ & $9(1$ & 12) & 0.8 \\
\hline Loop diuretics & $1052(59)$ & $1472(85)$ & $108(74)$ & $119(87)$ & 887 & (57) & $1290(85)$ & $57(71)$ & 63 & $(85)$ & $<0.001$ \\
\hline Thiazides & $199(11)$ & $125(7)$ & $24(17)$ & $14(10)$ & 166 & (11) & $104(7)$ & $9(11)$ & $7(1$ & 10) & 0.1 \\
\hline Loop diuretics + thiazides & $88(5)$ & $100(6)$ & $13(9)$ & $11(8)$ & 71( & & $82(5)$ & $4(5)$ & $7(9$ & & 0.07 \\
\hline
\end{tabular}

ACE angiotensin-angiotensin-converting enzyme inhibitors, ARB angiotensin receptor blockers, CKD-EPI Chronic kidney disease Epidemiology collaboration, GFR glomerular filtration rate, LVEF left ventricular ejection fraction, MRA mineralocorticoids receptors antagonists

\section{Predictors for dyskalemia}

The results of multivariate logistic regression to determine factors associated with the presence of dyskalemia on admission are detailed in Table 3.

A higher comorbidity burden (assessed by Charlson comorbidity index), diabetes mellitus, worse renal function, lower hemoglobin values, higher natremia, and the use of RAAS inhibitors and diuretics were independently associated with the presence of hyperkalemia on admission.

On the contrary, the previous history of HF-related hospitalization and higher intensity of diuretic treatment were independently related to the presence of hypokalemia on admission.

Regarding potassium levels before discharge, hyperkalemia was related to a higher Charlson index and low sodium levels, while hypokalemia was associated with the use of loop diuretics and thiazides. 
Table 2. Baseline characteristics according to serum potassium levels before discharge

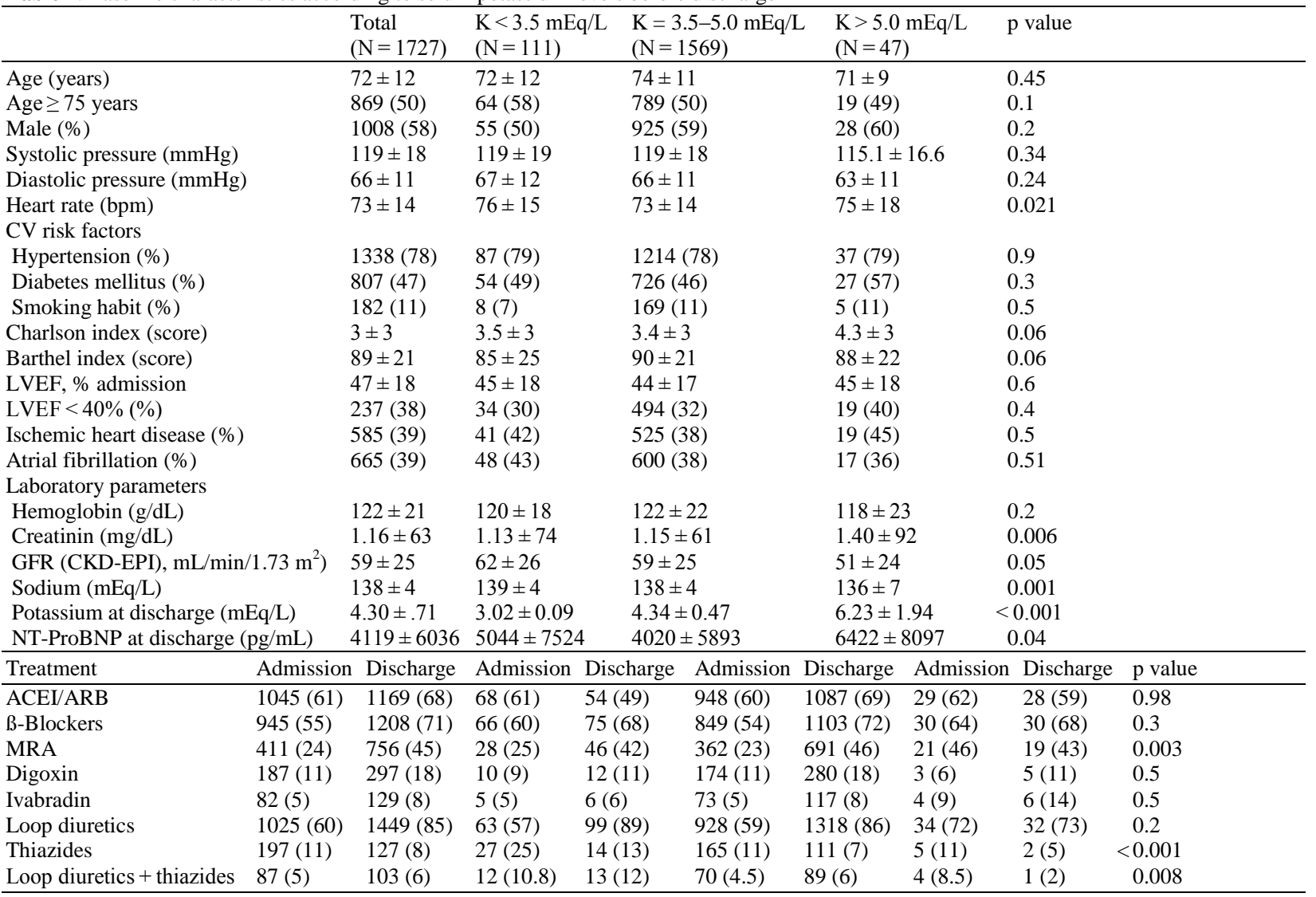

ACE angiotensin-angiotensin-converting enzyme inhibitors, ARB angiotensin receptor blockers, CKD-EPI chronic kidney disease epidemiology collaboration, GFR glomerular filtration rate, LVEF left ventricular ejection fraction, MRA mineralocorticoids receptors antagonists

Table 3. Univariate and multivariate analysis of predictors dyskalemia on admission

\begin{tabular}{|c|c|c|c|c|c|c|c|c|}
\hline & \multicolumn{4}{|c|}{ Hyperkalemia on admission } & \multicolumn{4}{|c|}{ Hypokalemia on admission } \\
\hline & \multicolumn{2}{|c|}{ Univariate } & \multicolumn{2}{|c|}{ Multivariate } & \multicolumn{2}{|c|}{ Univariate } & \multicolumn{2}{|c|}{ Multivariate } \\
\hline & $\beta$ & $\mathrm{p}$ value & $\beta$ & $\mathrm{p}$ value & $\beta$ & $\mathrm{p}$ value & $\beta$ & $\mathrm{p}$ value \\
\hline Age & 0.015 & 0.131 & & & -0.0046 & 0.510 & & \\
\hline Sex & -0.033 & 0.885 & & & -0.0695 & 0.691 & & \\
\hline Diabetes mellitus & 0.2400 & 0.016 & & & 0.1012 & 0.324 & & \\
\hline Previous HF admission & 0.0813 & 0.215 & & & 0.1185 & 0.014 & 0.1099 & 0.024 \\
\hline Charlson comorbidity index & 0.993 & $<0.001$ & & & 0.993 & $<0.001$ & & \\
\hline Systolic arterial pressure & -0.015 & 0.032 & & & -0.001 & 0.833 & & \\
\hline & & -0.005 & & & -0.0026 & 0.526 & & \\
\hline GFR (CKD-EPI), mL/min/1.73 $\mathrm{m}^{2}$ & -0.046 & $<0.001$ & -0.0482 & $<0.001$ & 0.0041 & 0.240 & & \\
\hline Serum sodium & -0.1028 & $<0.001$ & -0.0913 & 0.000 & 0.0098 & 0.614 & & \\
\hline ACEI/ARB & 0.9305 & 0.001 & 1.018 & $<0.001$ & 0.0219 & 0.902 & & \\
\hline MRA & 0.3666 & 0.001 & 0.3284 & 0.015 & 0.0422 & 0.728 & & \\
\hline Loop diuretics & 0.1923 & 0.041 & & & 0.1448 & 0.086 & & \\
\hline Loop diuretics + thiazides & -.0018 & 0.997 & & & 0.7164 & 0.022 & 0.6701 & 0.034 \\
\hline
\end{tabular}

ACE/ARB angiotensin angiotensin-converting enzyme inhibitors/angiotensin receptor blockers, CKD-EPI chronic kidney disease epidemiology collaboration, GFR glomerular filtration rate, HF heart failure, MRA mineralocorticoids receptors antagonists. 
In-hospital mortality was 3.9\% (69 patients). Among patients with hyperkalemia, the mortality rate was $12.3 \%$ (10 patients) and for patients with hypokalemia, 6.2\% (9 patients). The mortality rate for patients with normal serum potassium levels was $3.2 \%$ (50 patients).

Correlation of serum potassium levels with in-hospital mortality draws a "J"-shaped curve with a higher mortality risk for those values that deviate from the central values (Fig. 3). Among patients with hyperkalemia, mortality risk increases parallel to potassium levels ( $p$ value $=0.002)$. Thus, for a serum potassium increase from 4 to $5 \mathrm{mEq} / \mathrm{L}$ the OR for in-hospital mortality is 1.4 (95\% CI: 1.10-1.87; $\mathrm{p}=0.002)$ whereas for an increase from 5 to $6 \mathrm{mEq} / \mathrm{L}$ the OR is $2.27(95 \% \mathrm{CI}: 1.49-3.45 ; \mathrm{p}=0.002)$. Regarding hypokalemia, there is also a higher risk associated with a decrease from 3 to $2 \mathrm{mEq} / \mathrm{L}$ $(\mathrm{OR}=1.99,[95 \% \mathrm{CI}: 1.00-3.96] ; \mathrm{p}=0.050)$.

On multivariate Cox regression, hyperkalemia on admission showed to be an independent predictor for in-hospital mortality $(\mathrm{OR}=2.32$ [95\% CI: 1.04-5.21], $\mathrm{p}=0.041)$ while hypokalemia did not show a statistically significant effect on acute mortality $(\mathrm{OR}=2.06$ [95\% CI: 0.93-4.53], $\mathrm{p}=0.075)$ (Fig. 4). Multivariate model showed adequate discrimination (area under ROC curve was 0.76 [95\% confidence interval: $0.70-0.92]$ ) and calibration (Hosmer-Lemeshow statistic test-p $=0.99$ ).

Considering the "J-shaped" relation of serum potassium levels with in-hospital mortality, we also analyzed the prognostic impact of hypokalemia, defined as serum potassium $<4 \mathrm{mEq} / \mathrm{L}$. With this threshold, the association of hypokalemia with in-hospital mortality reached statistical signification (OR: 2.14 [95\% CI: $1.04-4.40] ; \mathrm{p}=0.038)$.

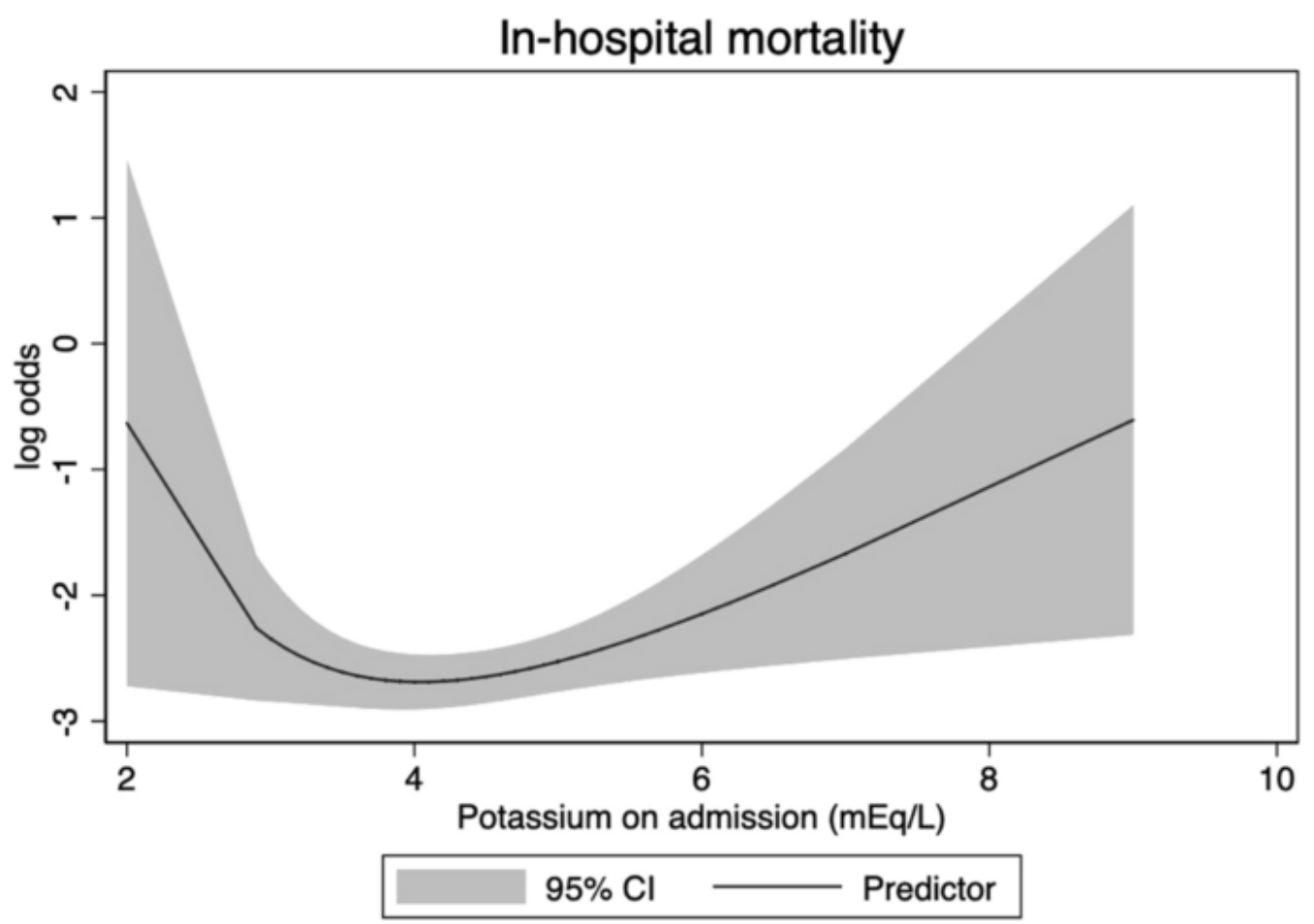

Fig. 3. Graphical representation of the association between serum potassium levels on admission and the odds ratio of in-hospital mortality. Asterisk represents a fractional polynomial model with four degrees of freedom ( -2 to 1$)$ and $95 \%$ CI indicates $95 \%$ confidence interval 
A total of $356(19.5 \%)$ patients had potassium level disturbances either on admission or before discharge: $122(6.7 \%)$ suffered hyperkalemia and $234(12.8 \%)$ hypokalemia. Serum potassium at discharge was $4.3 \pm 0.71 \mathrm{mEq} / \mathrm{L}$, which does not constitute a significant change when compared to admission values $(-0.009 \mathrm{mEq} / \mathrm{L}[95 \% \mathrm{CI}:-0.035$ to 0.053$] ; \mathrm{p}=0.696)$.

Regarding the changes in potassium levels during hospitalization, patients with hypokalemia and hyperkalemia on admission often had normalized potassium levels before discharge. Of a total of 139 patients with hypokalemia on admission in whom serum potassium levels were evaluated before discharge, 110 (79.2\%) had normalized serum potassium levels before discharge. Regarding the 72 patients presenting hyperkalemia on admission and in whom kalemia was recorded before discharge, 66 (89.2\%) showed normal serum potassium levels at discharge. Thus, only 27 patients (19.5\%) presented persistent hypokalemia at discharge and 6 patients (8.1\%) persistent hyperkalemia at discharge (Fig. 5).

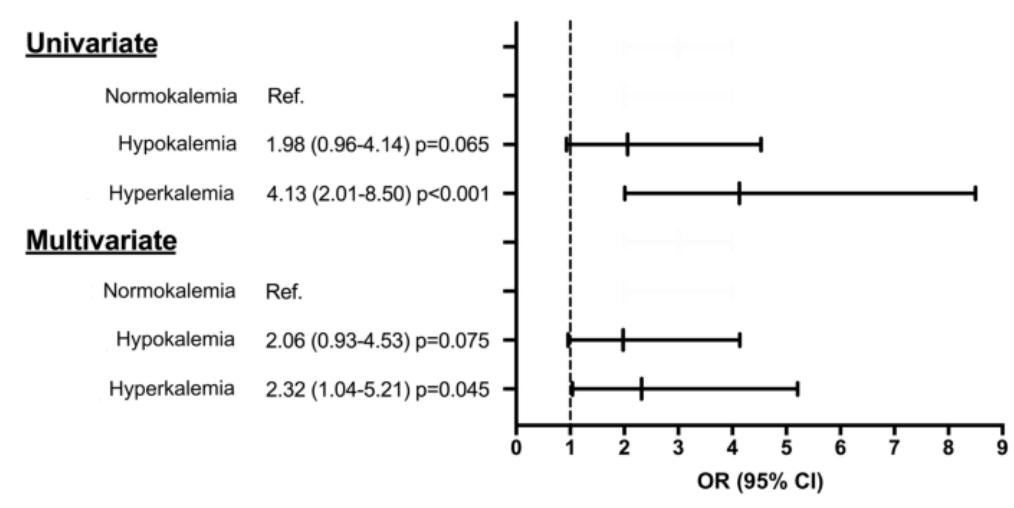

Fig. 4. Univariate and multivariate analysis of in-hospital mortality according to serum potassium levels on admission. OR: Odds ratio, 95\% CI: 95\% confidence interval. Asterisk represents the covariates included in the model were age, presence of previous admissions for HF, peripheral arterial disease, blood pressure, hemoglobin, natremia, GFR, NT-ProBNP levels, baseline functional class (NYHA), and Charlson and Barthel indices

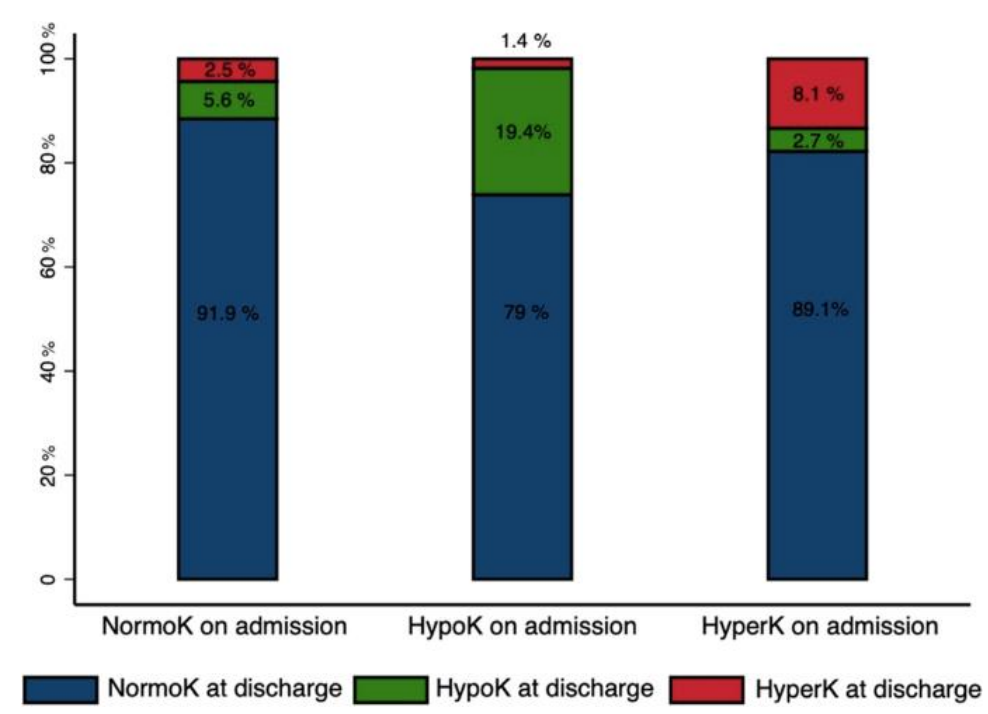

Fig. 5. Graphical representation of changes in serum potassium during hospitalization for patients with normokalemia, hypokalemia, and hyperkalemia on admission 
Figure 6 shows Kaplan-Meier 12-month mortality curves for the three predefined kalemia groups. In multivariate Cox regression analysis, the development of any dyskalemic pattern during admission was associated with a higher mortality risk at 12-month when compared to those patients who maintained normal serum potassium levels throughout the hospitalization period (hazard ratio $=1.48$ [95\% CI: $1.12-$ 1.96]; $\mathrm{p}=0.005)$. Dyskalemic patterns with the stronger association with mortality were persistent hypokalemia (hazard ratio $=3.17$ [95\% CI: 1.71-5.88; $\mathrm{p}<0.001$ ) and transient hyperkalemia (hazard ration $=1.75[95 \% \mathrm{CI}: 1.07-2.86] ; \mathrm{p}=0.023)($ Table 4$)$.

The multivariate mortality model showed adequate discrimination (Harrel's $\mathrm{C}$ statistic $=0.75$ ) and calibration (Gronnesby Borgan goodness-of-fit test $\mathrm{p}>0.1$ ).

A

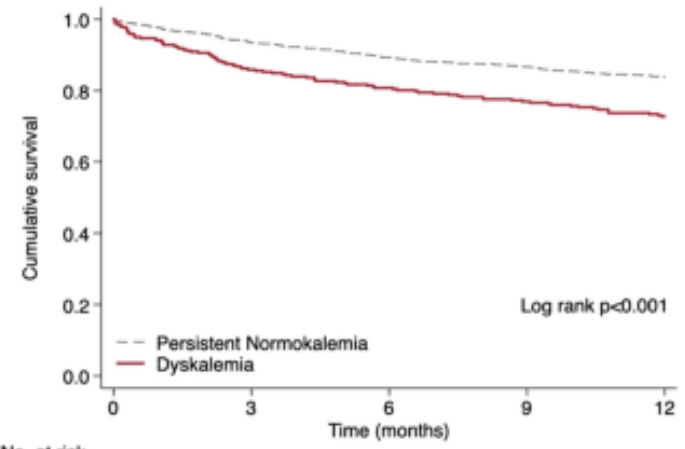

B

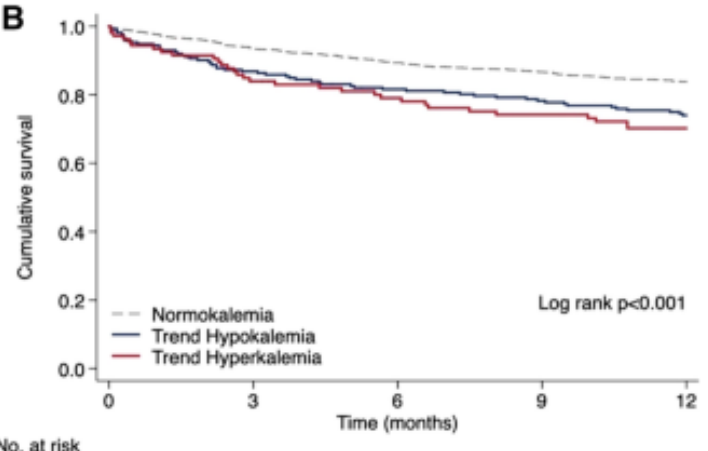

\section{No. at risk}

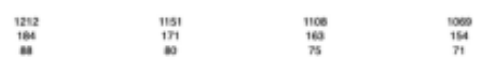

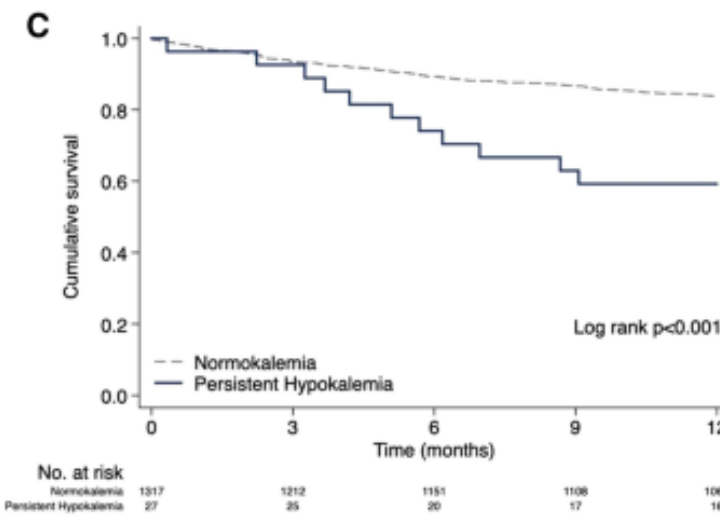

D
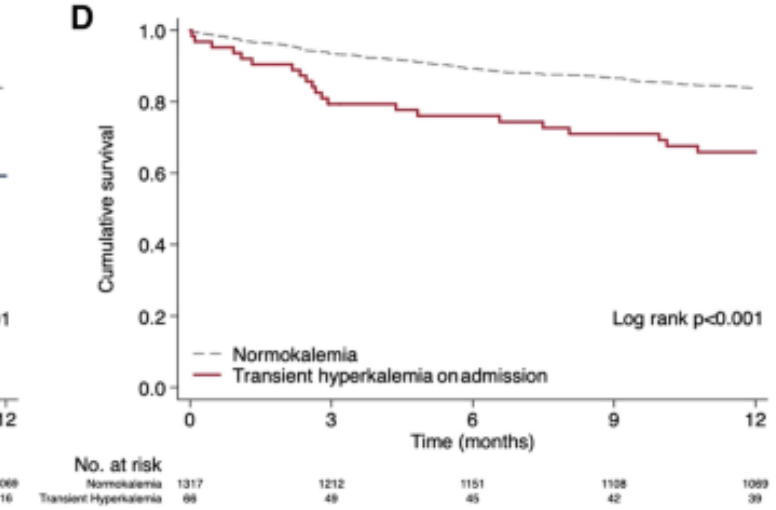

Fig. 6. Kaplan-Meier survival analysis for 12-month all-cause mortality depending on different dyskalemic patterns. a Persistent normokalemia versus any dyskalemic patter during hospitalization. b Persistent normokalemia versus hypokalemia versus hyperkalemia. c Persistent normokalemia versus persistent hypokalemia during hospitalization. d Persistent normokalemia versus transient hyperkalemia + The covariates evaluated were age, diabetes mellitus, previous diagnosis of HF, presence of prior admissions for HF, Charlson and Barthel comorbidity indices, blood pressure, hemoglobin, natremia, GFR, LVEF, signs of congestion at discharge, NYHA functional class at discharge, and treatment with RAAS inhibitors, loop diuretics, and thiazides at discharge. 
Table 4. Univariate and multivariate analysis of 12 months mortality according to dynamic changes of serum potassium levels during hospitalization.

\begin{tabular}{lllll}
\hline & Univariate & & Multivariate & \\
\hline & HR $(95 \%$ CI $)$ & p value & HR $(95 \%$ CI $)$ & p value \\
\hline K $>4$ & $1.60(1.28-2.00)$ & $<0.001$ & $1.26(0.98-1.64)$ & 0.070 \\
Dyskalemia & $1.83(1.43-2.36)$ & $<0.001$ & $1.48(1,12-1.96)$ & 0.005 \\
Persistent hypokalemia & $2.85(1.55-5.23)$ & 0.001 & $3.17(1.71-5.88)$ & $<0.001$ \\
Transient on-admission hyperkalemia & $2.41(1.54-3.78)$ & $<0.001$ & $2.27(1.07-2.86)$ & 0.023 \\
New onset hyperkalemia & $1.85(0.95-3.62)$ & 0.069 & $1.58(0.92-2.69)$ & 0.909 \\
New onset hypokalemia & $1.41(0.86-2.31)$ & 0.171 & $1.39(0.82-2.37)$ & 0.091 \\
Hyperkalemia at any point & $1.75(0.93-3.29)$ & 0.081 & $1.03(0.51-2.07)$ & 0.933 \\
Hypokalemia at any point & $1.74(1.29-2.34)$ & $<0.001$ & $1.63(1.17-2.26)$ & 0.003 \\
\hline
\end{tabular}

The covariates evaluated were age, diabetes mellitus, previous diagnosis of HF, presence of previous admissions for HF, Charlson and Barthel comorbidity indices, blood pressure, hemoglobin, natremia, GFR, LVEF, signs of congestion at discharge, NYHA functional class at discharge, and treatment with RAAS inhibitors, loop diuretics and thiazides at discharge

dyskalemia any potassium disturbance (hypo-hyperkalemia) on admission or at discharge, persistent hypokalemia hypokalemia on admission and at discharge, transient on-admission hyperkalemia hyperkalemia on admission and normokalemia at discharge, new onset hyperkalemia normokalemia on admission and hyperkalemia at discharge, new onset hypokalemia normokalemia on admission and hypokalemia at discharge, hyperkalemia at any point hyperkalemia on or at discharge, hypokalemia at any point hypokalemia on admission or at discharge

\section{Discussion}

\section{Principal findings}

In our cohort of AHF patients, $19.5 \%$ of patients presented dyskalemia at any point during the hospitalization period. The presence of hyperkalemia or hypokalemia, either on admission or before discharge, is related to a lower in-hospital and 12-month survival. On-admission hyperkalemia, but not hypokalemia, was independently associated with a higher risk of in-hospital mortality. Correlation between serum potassium levels and in-hospital mortality draws a "J-shaped" curve with a lower risk for those patients with normal kalemia.

Interestingly, we observed dynamic changes in kalemia throughout hospitalization, determining the appearance of specific evolutionary patterns. Potassium disorders present on admission are often transient. On the other hand, the disorders observed at discharge usually develop during hospitalization but are not present on admission. Thus, close monitoring of kalemia during hospitalization is paramount, since a normal value on admission does not exclude the appearance of new alterations. We also identified different evolutionary patterns of dyskalemia that are related to a worse prognosis during follow-up, such as persistent hypokalemia and hyperkalemia on admission normalized before discharge.

\section{Dyskalemia in acute heart failure}

The harmful effect of serum potassium levels disturbances in the setting of chronic heart failure is well-known [6, 12,13,14]. However, little is known about the prognostic impact of these alterations in the setting of AHF, either during the hospitalization period or in the long-term follow-up. Furthermore, available information was obtained from the sub-analysis of clinical trials with strict inclusion and exclusion criteria $[7,8,15]$ evaluating, therefore, a very selected population [9].

Formiga et al. [9] found an association between hyperkalemia on admission and the combined event of mortality and rehospitalization at 1-year, in a restricted population of patients older than 74 years. Tromp et al. [15] carried out a sub-analysis of the PROTECT trial, showing a linear association between serum potassium levels and mortality in patients with AHF. However, this was not confirmed in the multivariate analysis, and patients with hypokalemia were excluded. Nuñez et al. [6] found an association between potassium levels and mortality after a follow-up longer than 2 years in patients with a recent episode of AHF. However, in this study, patients were included just before discharge without evaluating the effect of these dyskalemic patterns and potassium dynamics throughout the hospitalization period. Last, Legrand et al. [16] studied the prognosis value of potassium on admission in a multicenter registry of AHD patients. In this study, hyperkalemia (defined as potassium $>4.5 \mathrm{mmol} / \mathrm{L}$ ) was associated with a higher risk of mortality at 90 days with no prognostic impact of hypokalemia. 
The significant heterogeneity of the above-described studies makes them difficult to compare, and consequently represent weak results. Conversely, our study describes the prevalence of potassium levels disorders during hospitalization for AHF, as well as its prognostic impact, in a prospective, multicenter, and contemporary cohort reflecting the real-life daily clinical practice.

\section{Prognostic impact of hyperkalemia}

In our cohort, the prevalence of hyperkalemia was $4.6 \%$ on admission and $2.7 \%$ at discharge, similar to that described in the literature $[6,9,15]$. Hyperkalemia on admission was associated with higher mortality at a 12-month follow-up in the multivariate analysis.

Different circumstances may explain the observed correlation between hyperkalemia on admission and higher mortality. First, hyperkalemia identifies a subgroup of high-risk patients, determined by older age, the highest comorbidity burden, and by the presence of high-risk markers such as a higher NTproBNP and lower hemoglobin levels. On the other hand, hyperkalemia is per se a cause of morbidity due to the rhythm disturbances, which may precipitate the appearance of potentially malignant arrhythmias.

However, the presence of hyperkalemia at discharge did not suppose a lower prescription of RAAS inhibitors. This fact highlights that the presence of hyperkalemia in a controlled environment does not prevent the use of drugs, and it may even be the consequence of its use.

\section{Prognostic impact of hypokalemia}

Hypokalemia is also a common complication in $\operatorname{HF}[7,9,15,17]$. In our cohort, hypokalemia on admission did not show to be related to higher in-hospital mortality when defined as serum potassium $<3.5 \mathrm{mEq} / \mathrm{L}$.

However, as potassium is a continuous variable with a "J"-shaped relation with adverse events, we analyzed the association of serum potassium levels $<4 \mathrm{mEq} / \mathrm{L}$ with in-hospital and long-term mortality [18]. With the $4 \mathrm{mEql} / \mathrm{L}$ threshold, this association reaches statistical signification for in-hospital mortality but loses it for long-term mortality. This may reflect a higher sensitivity of this threshold to identify those patients at a higher risk of events leading to in-hospital mortality. However, the ability to identify patients with poorer long-term prognosis is hampered with the use of the $4 \mathrm{mEq} / \mathrm{L}$ threshold. We hypothesize that although serum potassium levels $<4 \mathrm{mEq} / \mathrm{L}$ are associated with poorer outcomes, a kalemia $<3.5 \mathrm{mEq} / \mathrm{L}$ is more specific in the identification patients with a high-risk profile in the followup.

Last, persistent hypokalemia from admission to discharge was associated with higher 12-month mortality, which can be explained by a more severe HF episode, with a greater need for diuretics and a higher degree of hyperaldosteronism with the consequent more significant loss of potassium in the urine [19].

\section{Prognostic impact related to serum potassium dynamic changes}

An increased risk of mortality at 180 days in patients with a serum potassium decrease more significant than $5 \%$ or $0.3 \mathrm{mEq} / \mathrm{L}$ during hospitalization has been previously described [8]. However, our study is the first to describe different evolutionary patterns during hospitalization, identifying persistent hypokalemia and transient hyperkalemia on admission corrected before discharge, as those dyskalemia patterns related to a worse prognosis during the 12-month follow-up.

Our findings emphasize the importance of close monitoring of potassium levels. Whether the worst prognosis associated with dyskalemia is directly related to abnormal serum potassium levels or secondary to the worse clinical profile of these patients is unknown. Currently, ongoing clinical trials with novel potassium chelating drugs will help to solve this question (NCT03888066). 
REDINSCOR II registry is a prospective observational registry, with the limitations inherent to this kind of epidemiological study. Thus, the described findings should not be interpreted as causal or confirmatory but as hypothesis generators. The statistical analysis has been conducted adjusting for the main confounding variables based on a concise literature review. We do not know the influence of possible factors that have not been taken into account. Potassium levels and changes in treatment during hospitalization were not available, so the impact of these parameters is unknown.

\section{Conclusions}

Serum potassium levels disturbances are a common complication during AHF hospitalization. The presence of hyperkalemia on admission was an independent factor of in-hospital mortality. The levels of serum potassium do not remain stable throughout the hospitalization period presenting dynamic variations in the majority of patients. Persistent hypokalemia and transient hyperkalemia were associated with higher 12-month mortality.

These findings highlight the importance of monitoring potassium levels and paying particular attention to patients who develop certain disorders during hospitalization due to the increased risk of fatal clinical events that they may present in the short and long term.

\section{Abbreviations}

ACE: Angiotensin-converting enzyme

AHF: Acute heart failure

ARB: Angiotensin II receptor blocker

HF: Heart failure

MRA: Mineralocorticoid receptor antagonist

RAAS: Renin-angiotensin-aldosterone system

\section{Funding}

This work is funded by the Instituto de Salud Carlos III (Ministry of Economy, Industry, and Competitiveness) and co-funded by the European Regional Development Fund, through the CIBER in cardiovascular diseases (CB16/11/00502).

Availability of data and material.

Yes.

Code availability

Yes. 


\section{Ethics declarations}

\section{Conflicts of interest}

The authors have no conflict of interest to declare.

\section{Ethics approval}

Yes.

Consent to participate

Yes.

\section{Consent for publication}

Yes.

\section{References}

1. Sayago-Silva I, García-López F, Segovia-Cubero J (2013) Epidemiología de la insuficiencia cardiaca en España en los últimos 20 años. Rev Española Cardiol 66:649-656. https://doi.org/10.1016/j.recesp.2013.03.014

2. Mentz RJ, O'Connor CM (2016) Pathophysiology and clinical evaluation of acute heart failure. Nat Rev Cardiol 13:28-35. https://doi.org/10.1038/nrcardio.2015.134

3. Ponikowski P, Voors AA, Anker SD et al (2016) 2016 ESC Guidelines for the diagnosis and treatment of acute and chronic heart failure. Eur Heart J 37:2129-2200m. https://doi.org/10.1093/eurheartj/ehw128

4. Urso C, Brucculeri S, Caimi G (2015) Acid-base and electrolyte abnormalities in heart failure: pathophysiology and implications. Heart Fail Rev 20:493-503. https://doi.org/10.1007/s10741-015-9482-y

5. Komajda M, Anker SD, Cowie MR et al (2016) Physicians' adherence to guideline-recommended medications in heart failure with reduced ejection fraction: data from the QUALIFY global survey. Eur J Heart Fail 18:514522. https://doi.org/10.1002/ejhf.510

6. Núñez J, Bayés-Genís A, Zannad F et al (2018) Long-term potassium monitoring and dynamics in heart failure and risk of mortality. Circulation 137:1320-1330. https://doi.org/10.1161/CIRCULATIONAHA.117.030576

7. Khan SS, Campia U, Chioncel $\mathrm{O}$ et al (2015) Changes in serum potassium levels during hospitalization in patients with worsening heart failure and reduced ejection fraction (from the EVEREST trial). Am J Cardiol 115:790-796. https://doi.org/10.1016/j.amjcard.2014.12.045

8. Salah K, Pinto YM, Eurlings LW et al (2015) Serum potassium decline during hospitalization for acute decompensated heart failure is a predictor of 6-month mortality, independent of N-terminal pro-B-type natriuretic peptide levels: an individual patient data analysis. Am Heart J 170:531-542.e1. https://doi.org/10.1016/j.ahj.2015.06.003

9. Formiga F, Chivite D, Corbella X et al (2019) Influence of potassium levels on one-year outcomes in elderly patients with acute heart failure. Eur J Intern Med 60:24-30. https://doi.org/10.1016/j.ejim.2018.10.016

10. Haukoos JS, Newgard CD (2007) Advanced statistics: missing data in clinical research — part 1: an introduction and conceptual framework. Acad Emerg Med 14:662-668. https://doi.org/10.1197/j.aem.2006.11.037

11. Newgard CD, Haukoos JS (2007) Advanced statistics: missing data in clinical research-part 2: multiple imputation. Acad Emerg Med 14:669-678. https://doi.org/10.1197/j.aem.2006.11.038

12. Hoss S, Elizur Y, Luria D et al (2016) Serum potassium levels and outcome in patients with chronic heart failure. Am J Cardiol 118:1868-1874. https://doi.org/10.1016/j.amjcard.2016.08.078

13. Davidsen L, Aldahl M, Krogager ML et al (2017) Associations of serum potassium levels with mortality in chronic heart failure patients. Eur Heart J 38:2890-2896. https://doi.org/10.1093/eurheartj/ehx460

14. Crespo-Leiro MG, Barge-Caballero E, Segovia-Cubero J et al (2019) Hyperkalemia in heart failure patients in Spain and its impact on guideline- directed medical therapy. Rev Esp Cardiol. https://doi.org/10.1016/j.recesp.2019.05.004

15. Tromp J, ter Maaten JM, Damman K et al (2017) Serum potassium levels and outcome in acute heart failure (data from the PROTECT and COACH trials). Am J Cardiol 119:290-296. https://doi.org/10.1016/j.amjcard.2016.09.038 
16. Legrand M, Ludes PO, Massy Z et al (2018) Association between hypo- and hyperkalemia and outcome in acute heart failure patients: the role of medications. Clin Res Cardiol 107:214-221. https://doi.org/10.1007/s00392-017-1173-3

17. Bielecka-Dabrowa A, Mikhailidis DP, Jones L et al (2012) The meaning of hypokalemia in heart failure. Int J Cardiol 158:12-17. https://doi.org/10.1016/j.ijcard.2011.06.121

18. Ferreira JP, Butler J, Rossignol P et al (2020) Abnormalities of potassium in heart failure: JACC state-of-the-art review. J Am Coll Cardiol 75:2836-2850. https://doi.org/10.1016/j.jacc.2020.04.021

19 .Girerd N, Pang PS, Swedberg K et al (2013) Serum aldosterone is associated with mortality and rehospitalization in patients with reduced ejection fraction hospitalized for acute heart failure: analysis from the EVEREST trial. Eur J Heart Fail 15:1228-1235. https://doi.org/10.1093/eurjhf/hft100 